\title{
Talking hypotheses
}

Andreas Ziegler, Inke König

Wiley-VCH, Weinheim, Germany, 2006. ISBN-13: 978-3527312528

..........

Bertram Müller-Myhsok

European Journal of Human Genetics (2007) 15, 817;

doi:10.1038/sj.ejhg.5201853

A

ndreas Ziegler and Inke $\mathrm{R}$ König have undertaken the daunting task to give an overview of current concepts (and applications) in genetic epidemiology. After an introductory section on (human) molecular genetics, including an important and very laudable section on data quality, they cover linkage and association in a thoughtfully selected, very complete way.
What is truly remarkable about this book is that it indeed does what it promises to do in the title. The book gives statistical details behind the methodology, without going into too much or too little detail. This makes it a long-sought addition to the literature. In every chapter there are questions and answers, which are very useful for self-study. To put the book to the test, I asked some of my students to read the book and give me their opinion, which was very positive in all cases. This is remarkable, as their respective backgrounds are partly from biology and partly from statistics. This argues that the book should indeed appeal to readers from all backgrounds, if only they are interested in the topic covered.

The text itself is very nicely written and easy to read. It is organised in mostly a question and answer style, which facilitates retrieval of the relevant information. A special feature is that many algorithms are presented in a kind of natural computer language, making them easy to understand and to program. There is also a (comparatively short) list of errata at http://www.imbs.uni-luebeck.de/ monogr.html.

All in all, it is easy to give a very strong recommendation for this book, and I should expect it to become the standard for its kind

Bertram Müller-Myhsok is at MPI Psychiatry, Munich, Germany. E-mail:bmm@mpipsykl.mpg.de 